\title{
1 Bioelectroanalysis in a Drop: Construction of a Glucose Biosensor
}

\author{
2 O. Amor-Gutiérrez, E. C. Rama,* M. T. Fernández-Abedul, and A. Costa-García*๑ \\ 3 Departamento de Química Física y Analítica, Universidad de Oviedo, Julián Clavería 8, 33006 Oviedo, Spain \\ 4 S Supporting Information
}

\begin{abstract}
This lab experiment describes a complete method to fabricate an enzymatic glucose electroanalytical biosensor by students. Using miniaturized and disposable screen-printed electrodes (SPEs), students learn how to use them as transducers and understand the importance SPEs have acquired in sensor development during the last years. Students can also revise concepts related to enzymatic assays, with glucose oxidase and horseradish peroxidase involved in subsequent reactions. Moreover, they learn the trends that current analytical chemistry follows presently such as miniaturization, portability, and low cost. At the same time, this experiment serves to teach basic analytical concepts (accuracy, precision, sensitivity, and selectivity) in a practical

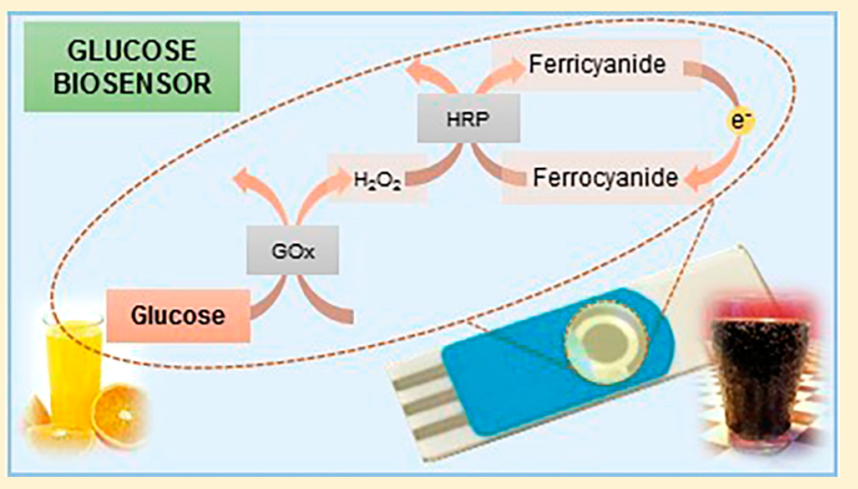
way. The high clinical interest of glucose, due to a large number of diabetes patients around the world, and the application of the sensor to analysis of real food samples make this experiment very attractive to students. The questions set out along this experiment help students to acquire skills for solving analytical problems from the very beginning.

KEYWORDS: Upper-Division Undergraduate, Analytical Chemistry, Laboratory Instruction, Hands-On Learning/Manipulatives, Quantitative Analysis, Electrochemistry, Bioanalytical Chemistry, Biotechnology, Carbohydrates, Enzymes
\end{abstract}

${ }_{24}^{23} \mathrm{M}_{\mathrm{t}}$ iniaturization is presently one of the most important trends in analytical chemistry. The reduction of the size 25 of analytical systems ${ }^{1}$ involves their simplification as well as a 26 decrease in costs, reagents, and sample volume. Furthermore, it 27 is related to many principles of Green Analytical Chemistry. ${ }^{2}$ 28 Electrochemical detection closely connects with these aims 29 because of its inherent ease of miniaturization. Moreover, an 30 improvement in productivity-related properties such as analysis 31 time or cost as well as in others related to environmental 32 benefits like waste production or energy consumption is very 33 advantageous. Other basic analytical properties (e.g., accuracy, 34 precision, sensitivity, and selectivity) are generally not 35 compromised since electrochemical analyis is among the most 36 sensitive detection techniques (as demonstrated by its leading 37 use in biosensing $)^{3}$ and mass production increases the precision 38 of disposable devices.

39 During the past few years, screen-printing technology has 40 been increasingly used in the fabrication of low-cost thick-film 41 electrodes with small size and good analytical characteristics. 42 During the last years, they have been the basis of many 43 biosensors $^{4-6}$ because of their low cost and simplicity. Another 44 advantage of screen-printed electrodes (SPEs) is the possibility 45 of doing in situ analysis. ${ }^{7}$ Apart from the electrodes, 46 electrochemical equipment (potentiostats) is also being 47 miniaturized.

48 The aim of this experiment is to build an enzymatic 49 electrochemical biosensor to measure glucose in real food 50 samples. Biosensing is a field that is growing continuously, as 51 demonstrated by the leading place of the journal Biosensors \&
Bioelectronics. ${ }^{8}$ Glucose is probably one of the most important 52 biological compounds because of its engagement in a multitude 53 of reactions. ${ }^{9}$ Glucose analysis in blood is very important and 54 common because of diabetes mellitus, a disease that is suffered 55 by approximately 150 million people around the world. ${ }^{10,11} 56$ This disease is produced when the pancreas does not generate 57 enough insulin or when the body cannot use the insulin it 58 produces in an effective way. This leads to an increased level of 59 glucose in the blood. Thus, determination of glucose is one of 60 the most important analytical problems in food science and 61 clinical analysis, so much so that glucose biosensors account for 62 approximately $85 \%$ of the entire biosensor market. ${ }^{12,13} 63$

In this experiment, the students develop an amperometric 64 glucose sensor using the bienzymatic system glucose oxidase 65 $(\mathrm{GOx}) /$ horseradish peroxidase (HRP) and ferrocyanide as an 66 electron-transfer mediator. ${ }^{14,15}$ Moreover, since the sensor is 67 fabricated using screen-printed carbon electrodes (SPCEs), 68 students are introduced to the miniaturization of analytical 69 devices.

The fabrication of this glucose sensor is based on a very 71 simple procedure reported by our research group, ${ }^{14,16,17}$ and it 72 is addressed to undergraduate students of advanced analytical 73 chemistry. The high educational content related to biosensor 74 principles and new contemporary trends in analytical chemistry 75

Received: December 9, 2016

Revised: $\quad$ March 5, 2017 
76 also makes this experiment very attractive for the training of 77 chemistry, biotechnology, or biochemistry students.

78 There are many laboratory experiments about detection of 79 glucose using the enzyme GOx, but to the best of our 80 knowledge this is the first undergraduate lab experiment that 81 uses screen-printed electrodes to develop a glucose sensor. 82 Moreover, the combination of a simple procedure with the use 83 of SPCEs eliminates time-consuming maintenance of conven84 tional electrodes commonly required with other reported 85 sensors. ${ }^{18-20}$

86 This laboratory experiment is very useful to introduce 87 students to both electrochemical and biosensor methodologies 88 and provides students with several objectives:

89 - Learn the principles of important electrochemical 90 techniques such as cyclic voltammetry and chronoamper91 ometry.

92 - Use low-cost, disposable, and miniaturized electrodes.

93 - Fabricate a glucose biosensor, optimize the parameters 94 influencing the analytical signal, and study the analytical 95 characteristics of the methodology.

96 - Analyze real food samples and learn how to validate the 97 methodology.

\section{EXPERIMENTAL SECTION}

99 This lab experiment is designed for a maximum of 15 100 undergraduate or Master's students working in groups of 101 three during four sessions of $4 \mathrm{~h}$ (the lab experiment planning 102 is more detailed in the Supporting Information). The 103 laboratory experiment consists of the following steps:

104 (i) Evaluation of the ferro/ferricyanide system using cyclic voltammetry to set the detection potential.

(ii) Optimization of the concentrations of enzymes and mediator.

(iii) Calibration of the biosensor and evaluation of the sensitivity.

(iv) Study of the precision.

(v) Evaluation of the selectivity.

(vi) Determination of glucose in real food samples.

\section{Instrumentation}

114 Electrochemical measurements were carried out with a $115 \mu$ AUTOLAB potentiostat (Metrohm, Switzerland) interfaced 116 with a computer system and controlled by Autolab GPES 4.9 117 software. Commercial screen-printed carbon electrodes (ref. 118 DRP-110; Figure 1) and the connector to the potentiostat (ref.

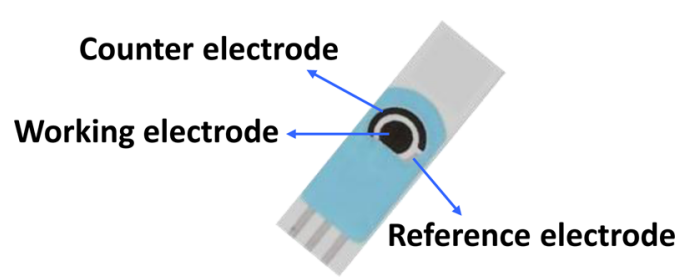

Figure 1. Picture of a screen-printed carbon electrode.

119 DRP-DSC) were purchased from DropSens (Spain). More 120 information on SPEs can be found in the student handout in 121 the Supporting Information.

122 Sensor Phase

123 The biosensor constructed in this experiment has a bienzymatic 124 sensor phase consisting of glucose oxidase and horseradish peroxidase, with ferrocyanide as an electron-transfer mediator. 125 The combination of these enzymes produces an enzymatic 126 cascade of reactions in which GOx and HRP are catalytically 127 linked. $^{21}$ These types of cascade schemes may produce signal 128 amplification and therefore enhance the sensitivity of the 129 biosensor. Another advantage is that by removal of the 130 hydrogen peroxide $\left(\mathrm{H}_{2} \mathrm{O}_{2}\right)$ generated, peroxide-induced 131 degradation of the GOx enzyme could be reduced. ${ }^{22}$ On the 132 other hand, redox mediators are frequently employed in 133 bienzymatic sensors because of their lower detection 134 potentials. ${ }^{10,23,24}$ This is very interesting since it improves the 135 selectivity: at lower potentials (in absolute value), fewer 136 compounds present in the sample are exposed to being 137 oxidized or reduced.

In the proposed enzymatic cycle, GOx catalyzes the oxidation 139 of glucose by oxygen, generating gluconic acid and $\mathrm{H}_{2} \mathrm{O}_{2}$. Then 140 HRP catalyzes the oxidation of ferrocyanide to ferricyanide, 141 consuming the $\mathrm{H}_{2} \mathrm{O}_{2}$ previously generated. The analytical signal 142 is the current intensity due to the electrochemical reduction of 143 the enzymatically generated ferricyanide. Scheme 1 shows the $144 \mathrm{~s} 1$

Scheme 1. Diagram of the Catalytic Reactions and the Reduction of Ferricyanide Produced on the Electrode Surface, Where GOx, HRP, and Ferrocyanide Are Immobilized $^{a}$

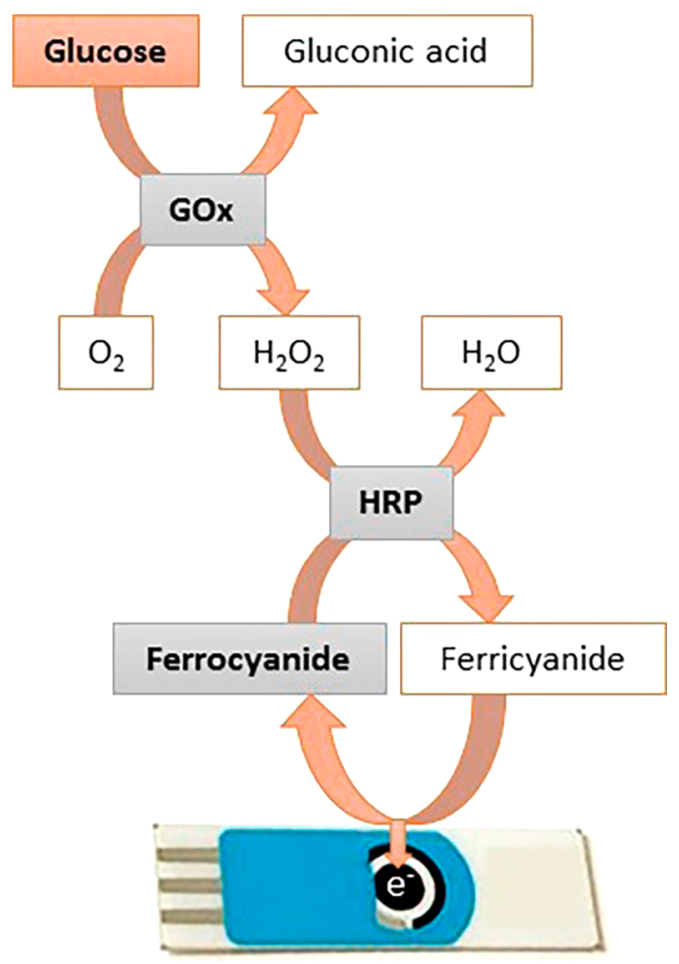

${ }^{a}$ Adapted with permission from ref 26. Copyright 2016 Elsevier.

reactions involved. Since glucose produces hydrogen peroxide 145 stoichiometrically, and this in turn produces ferricyanide, the 146 concentration of glucose in the measuring solution can be 147 calculated by measuring the concentration of reduced 148 ferricyanide.

Procedure

Students prepare the biosensors by depositing onto the surface 151 of the working electrode $10 \mu \mathrm{L}$ of a mixture containing the 152 enzymes and the mediator at the adequate concentrations, 153 
154 prepared in a $0.1 \mathrm{M}$ Tris- $\mathrm{HNO}_{3}$ buffer $(\mathrm{pH}$ 7.0). Then, after a 155 drying step at room temperature (approximately 40-60 $\mathrm{min}$ ), 156 the sensors are ready to use. If they are going to be employed in 157 the following days or weeks, they must be kept protected from 158 light and at $4{ }^{\circ} \mathrm{C}$.

159 All of the measurements are carried out at room temperature 160 with all three electrodes of the SPCE (working, counter, and 161 pseudoreference) covered with $40 \mu \mathrm{L}$ of the measuring 162 solution.

163 The analytical signal is the current intensity measured after 164 recording a chronoamperogram (current vs time) at a potential 165 of $-0.1 \mathrm{~V}$ vs Ag pseudoreference electrode for $100 \mathrm{~s}$ (Figure 2).

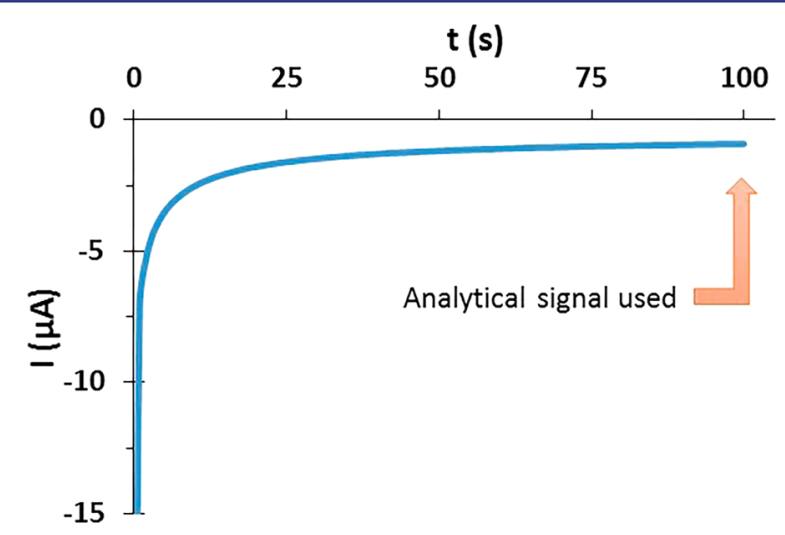

Figure 2. Chronoamperogram obtained at $-0.1 \mathrm{~V}$ vs $\mathrm{Ag}$ pseudoreference electrode, recorded in a $0.5 \mathrm{mM}$ glucose solution with 1.6 units $/ \mu \mathrm{L}$ GOx, 2.5 units $/ \mu \mathrm{L} \mathrm{HRP}$, and $0.05 \mathrm{M}$ ferrocyanide (in $0.1 \mathrm{M}$ Tris- $\mathrm{HNO}_{3}$ buffer $(\mathrm{pH} 7.0)$ ) immobilized on the working electrode $(10 \mu \mathrm{L})$.

166 A negative current due to the reduction of ferricyanide is 167 obtained, as shown in Figure 2. SPCEs are considered as 168 disposable, and a different sensor is used for each measurement.

\section{HAZARDS}

170 Nitric acid, used for the preparation of the Tris- $\mathrm{HNO}_{3}$ buffer, is 171 corrosive and causes serious burns, so it must be handled with 172 appropriate gloves, safety glasses, and protective clothing. The 173 main hazard of potassium ferrocyanide is that it releases a very 174 toxic gas when it is in contact with acids.

\section{${ }_{175} \square$ RESULTS}

\section{Electrochemical Behavior of Ferrocyanide}

177 After learning about the cascade of enzymatic reactions, 178 students knew that they had to measure the current intensity 179 due to the reduction of ferricyanide. Thus, a potential for 180 ferricyanide reduction had to be applied. Then students 181 investigated the process of the ferro/ferricyanide system, 182 recording a cyclic voltammogram $(\mathrm{CV}$ ) (one $\mathrm{CV}$ can be 183 recorded by each group) in a drop of $0.01 \mathrm{M}$ ferrocyanide 184 solution from -0.2 to $0.8 \mathrm{~V}$ at a scan rate of $50 \mathrm{mV} / \mathrm{s}$ (Figure 185 3). Previously, a CV was recorded in the background electrolyte 186 to confirm that there was no interference in the potential 187 window scanned. Ferrocyanide shows an electrochemical 188 process according to the following reaction:

$$
\left[\mathrm{Fe}(\mathrm{CN})_{6}\right]^{4-} \rightleftarrows\left[\mathrm{Fe}(\mathrm{CN})_{6}\right]^{3-}+\mathrm{e}^{-}
$$

189 Looking at the voltammogram, students discussed the 190 reversibility of the process. In this case, the system was

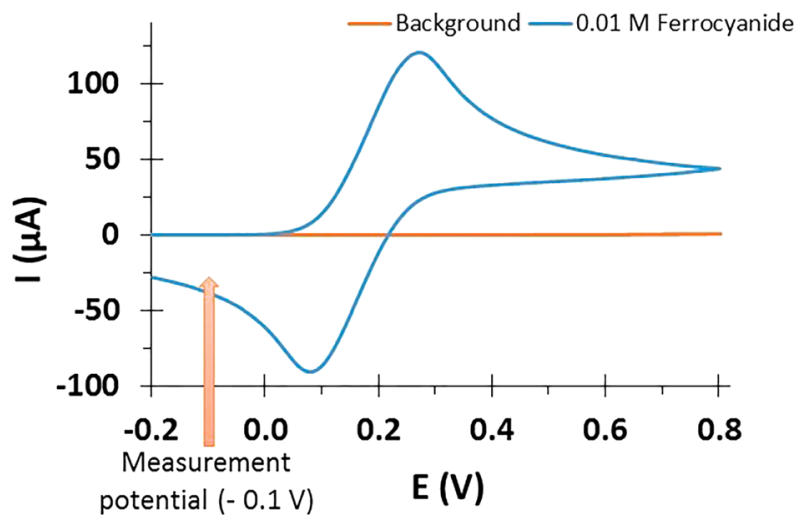

Figure 3. Cyclic voltammogram for $0.1 \mathrm{M}$ Tris- $-\mathrm{HNO}_{3}$ buffer solution ( $\mathrm{pH}$ 7.0) (background, orange) and for $0.01 \mathrm{M}$ ferrocyanide (blue).

considered to be quasireversible since the difference in 191 potentials is $183 \mathrm{mV}$ and the ratio of peak currents is $1.1 .^{25} 192$

To set the adequate potential for recording the chronoam- 193 perograms used for glucose determination, students looked at 194 the process recorded in the $\mathrm{CV}$ for the ferrocyanide solution 195 (Figure 3) and chose the potential they considered better $(-0.1196$ $\mathrm{V}$ in this case). They argued that at this potential ferricyanide 197 can be reduced to obtain the initial product, ferrocyanide, 198 which is maintained as a complex of Fe(II) on the electrode, 199 and therefore, all of the current intensity comes from the 200 reduction of the ferricyanide (Fe(III)) enzymatically produced. 201 Thus, higher glucose concentrations produce higher current 202 intensities (in absolute value).

Optimization of the Enzyme Concentrations

203

Students had to know that introducing an analytical method- 205 ology requires that once the analytical signal has been 206 identified, the different variables involved must be optimized 207 before the analytical properties (sensitivity, precision, etc.) can 208 be studied. Then students were requested to identify which 209 were the different variables that can influence the signal. After 210 discussion, variables such as $\mathrm{pH}$, electrolyte, and enzyme and 211 mediator concentrations were mentioned. The instructor 212 explained that a $0.1 \mathrm{M}$ Tris- $\mathrm{HNO}_{3}$ buffer $(\mathrm{pH}$ 7.0) was chosen 213 since it was used for similar reported glucose sensors. ${ }^{14,26}$ Thus, 214 the enzyme and ferrocyanide concentrations were identified as 215 relevant variables that should be optimized. With this aim, 216 students prepared sensors with various concentrations of the 217 enzymes, using $10 \mu \mathrm{L}$ of mixtures with different concentrations 218 of the enzymes and a constant concentration of ferrocyanide. 219 For each mixture, chronoamperograms were recorded in the 220 background electrolyte (buffer solution) and in a $0.4 \mathrm{mM} 221$ glucose solution.

Figure 4 shows results the students obtained for the different $223 \mathrm{f} 4$ concentrations of enzymes studied. It can be noted that the 224 intensities for glucose solutions were very similar for all of the 225 mixtures, whereas the intensities for the background increased 226 with the concentration of HRP, with the lowest obtained for 2.5227 units/ $\mu \mathrm{L}$ HRP. For this concentration of HRP, the lowest 228 background was obtained for 1.6 units/ $\mu \mathrm{L}$ GOx. Therefore, 229 students chose those enzyme concentrations for the con- 230 struction of the biosensor.

The following step was the optimization of the concentration of 233 ferrocyanide. Different concentrations of the electron-transfer 234 mediator $(0.05,0.1$, and $0.2 \mathrm{M})$ were studied using the enzyme 235 


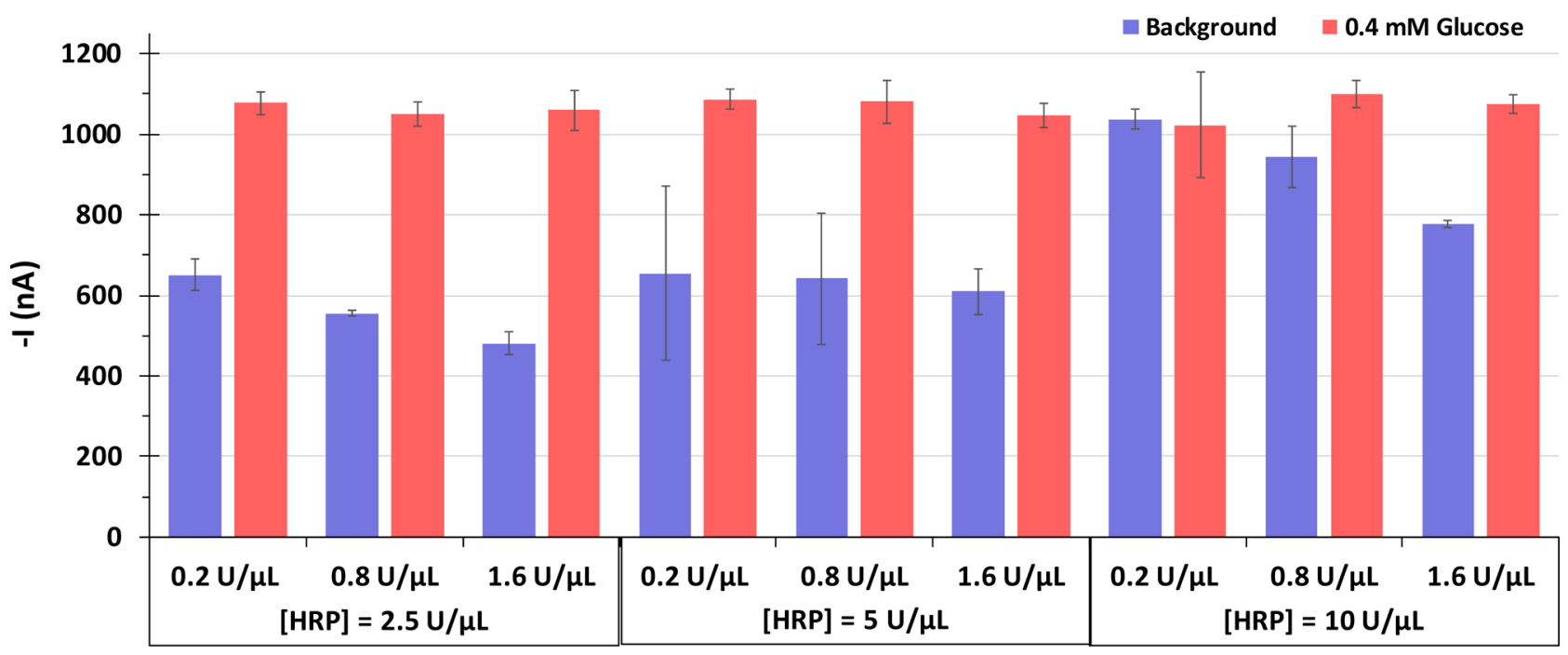

Figure 4. Current intensities recorded at $-0.1 \mathrm{~V}$ vs $\mathrm{Ag}$ pseudoreference electrode after $100 \mathrm{~s}$ in buffer solution (background, blue) and a $0.4 \mathrm{mM}$ glucose solution $(\mathrm{red})$. $[\mathrm{HRP}]=2.5,5$, or 10 units $/ \mu \mathrm{L} ;[\mathrm{GOx}]=0.2,0.8$, or 1.6 units $/ \mu \mathrm{L}$; [ferrocyanide] $=0.1 \mathrm{M}$. Data are given as mean \pm standard deviation $(\mathrm{SD})(n=3)$.

236 concentrations optimized in the previous section $(1.6$ units $/ \mu \mathrm{L}$ $237 \mathrm{GOx}$ and 2.5 units/ $\mu \mathrm{L}$ HRP).

238 Figure 5 presents the current intensities obtained by the 239 students using different concentrations of ferrocyanide. As can

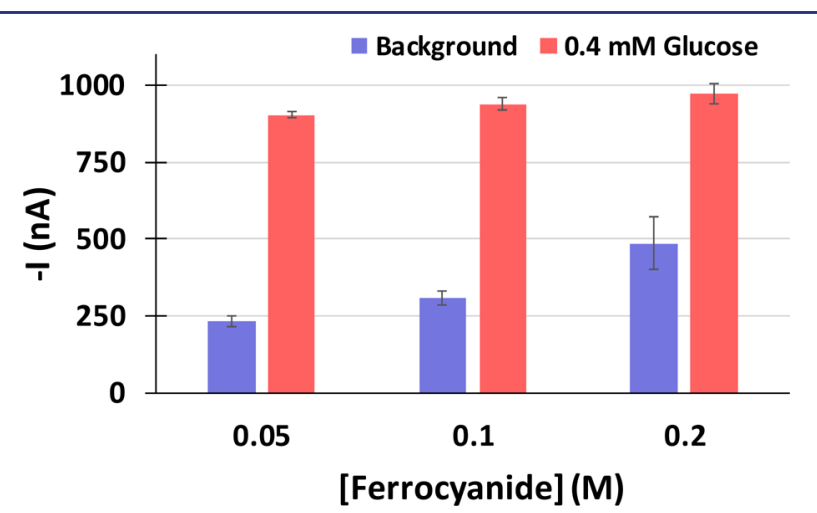

Figure 5. Current intensities recorded at $-0.1 \mathrm{~V}$ vs $\mathrm{Ag}$ pseudoreference electrode after $100 \mathrm{~s}$ in buffer solution (background, blue) and $0.4 \mathrm{mM}$ glucose solution (red) with ferrocyanide concentrations of $0.05,0.1$, and $0.2 \mathrm{M}$ using 1.6 units/ $\mu \mathrm{L}$ GOx and 2.5 units/ $\mu \mathrm{L}$ HRP (deposition of a $10 \mu \mathrm{L}$ drop). Data are given as mean $\pm \operatorname{SD}(n=3)$.

240 be seen, the analytical signal for a $0.4 \mathrm{mM}$ glucose solution 241 increased very slightly with the ferrocyanide concentration. 242 However, the intensities in the background decreased when the 243 concentration of the mediator was reduced. Therefore, students 244 concluded that $0.05 \mathrm{M}$ ferrocyanide was the best concentration 245 because it gave a higher signal-to-noise ratio.

\section{Calibration of the Biosensor}

247 Once the sensor phase had been optimized, students carried 248 out a calibration plot in order to know how the biosensor 249 responded to increasing glucose concentration and to revise 250 some analytical characteristics of the methodology, namely, 251 capital (e.g., accuracy, representativeness), basic (e.g., sensi252 tivity, precision), and productivity-related properties (e.g., 253 analysis time and cost). As shown in Figure 6, they found a 254 linear relationship between the current intensity and glucose

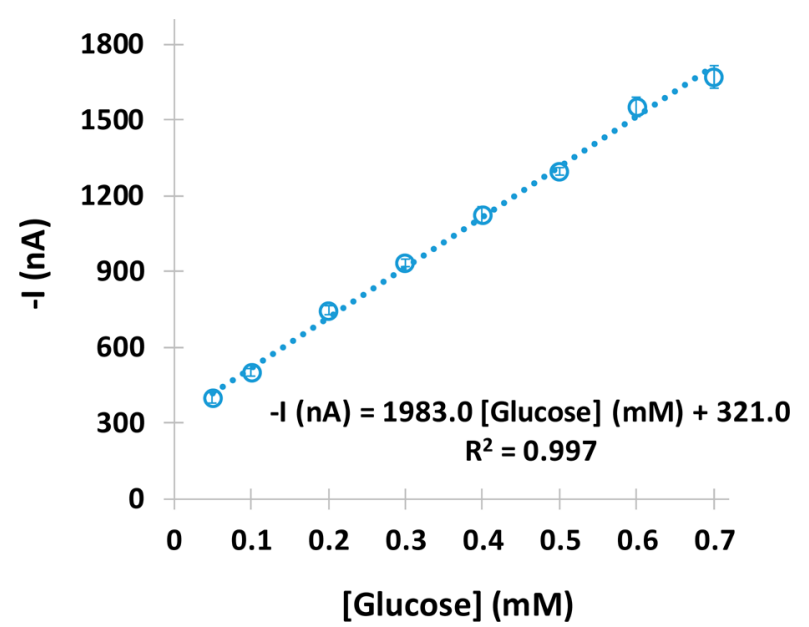

Figure 6. Calibration plot obtained with the glucose biosensor fabricated by immobilizing $10 \mu \mathrm{L}$ of 1.6 units $/ \mu \mathrm{L}$ GOx, 2.5 units $/ \mu \mathrm{L}$ $\mathrm{HRP}$, and $0.05 \mathrm{M}$ ferrocyanide solution. Data are given as mean $\pm \mathrm{SD}$ $(n=3)$.

concentration in the range between 0.05 and $0.7 \mathrm{mM}$ with a 255 sensitivity of $1983.0 \mathrm{nA} / \mathrm{mM}$. Students also calculated 256 important parameters such as the limit of detection (LOD) 257 and the limit of quantification (LOQ) according to the 258 following equations: $\mathrm{LOD}=3 s_{b} / m$ and LOQ $=10 s_{b} / m$, where 259 $m$ is the slope of the calibration curve and $s_{b}$ is the standard 260 deviation of the intercept. ${ }^{27,28}$ For this biosensor, the LOD and 261 LOQ values thus calculated were 0.03 and $0.1 \mathrm{mM}, 262$ respectively. They were also motivated to discuss other glucose 263 sensors found in the literature ${ }^{14,22,26,29,30}$ and to compare their 264 analytical characteristics (sensitivity, precision, linear range, 265 LOD, and LOQ) as well as the procedure of construction 266 (simplicity and fabrication time).

Precision

267

268

The precision of the calibration curve is very important, 269 especially when dealing with an analyte as important as glucose. 270 In order to evaluate it, three calibration curves measured on 271 different days by different groups of students using different 272 solutions were compared (data are shown in Table 1). They $273 \mathrm{tl}$ 
Table 1. Equations of the Calibration Plots for Three Glucose Biosensor Series

\begin{tabular}{ccccc}
$\begin{array}{c}\text { Calibration } \\
\text { Curve }\end{array}$ & $\begin{array}{c}\text { Slope, } \\
\mathrm{nA} / \mathrm{mM}^{a}\end{array}$ & $\begin{array}{c}\text { Intercept, } \\
\mathrm{nA}^{b}\end{array}$ & $R^{2}$ & $\begin{array}{c}\text { Linear Range, } \\
\mathrm{mM}\end{array}$ \\
1 & 1983.0 & 321.0 & 0.996 & $0.05-0.70$ \\
2 & 2136.0 & 168.0 & 0.997 & $0.05-0.70$ \\
3 & 2105.0 & 230.0 & 0.991 & $0.05-0.70$ \\
${ }^{a}$ Mean $\pm \mathrm{SD}=2080 \pm 81$. & ${ }^{b}$ Mean $\pm \mathrm{SD}=240 \pm 77$. & \\
\hline
\end{tabular}

274 showed very good reproducibility, with a relative standard 275 deviation of the slopes of $3.9 \%(n=3)$. In this way, students 276 understood that a biosensor must present good reproducibility 277 notwithstanding the fabrication day, the day of use, and the 278 operator. Since the temperature is not controlled (experiments 279 were done at room temperature), this was also indicative of the 280 robustness of the methodology.

\section{Selectivity}

282 Selectivity is another important property of a biosensor that has 283 to be taken into account. Students evaluated how the presence 284 of some species affected the analytical signal. In this case, 285 fructose and ascorbic acid were chosen as possible interferences 286 that could be found in real samples. Thus, different biosensors 287 were constructed for determination in mixtures of glucose/ 288 fructose and glucose/ascorbic acid. The results obtained are 289 reported in Table 2.

Table 2. Study of Fructose and Ascorbic Acid Interferences in the Glucose Sensor

$\begin{array}{ccc}\text { Sample } & -\mathrm{I}, \mathrm{nA} & \pm \mathrm{SD}, \mathrm{nA}^{b} \\ \text { Background } & 386 & 11 \\ \text { Glucose 0.3 mM } & 791 & 21 \\ \text { Glucose 0.3 mM/Fructose } 0.3 \mathrm{mM} & 852 & 30 \\ \text { Glucose 0.3 mM/Ascorbic Acid } 0.3 \mathrm{mM} & 538 & 25 \\ \text { Glucose 0.3 mM/Ascorbic Acid } 6 \mu \mathrm{M}^{a} & 829 & 29 \\ { }^{a} \text { Ratio in real samples. }{ }^{b} n=3 . & & \\ \end{array}$

290 As can be seen in Table 2, fructose and ascorbic acid 291 produced the opposite effect on the analytical signal. In the case 292 of fructose, a slight increase in the signal was seen; meanwhile, 293 ascorbic acid (usually employed as an antioxidant) produced a 294 decrease in the signal. The effect is not so important when the 295 glucose:ascorbic acid ratio is similar to that found in real 296 samples (e.g., orange juice ${ }^{31}$ ). Students were encouraged to 297 look for possible solutions to avoid the interference produced 298 by ascorbic acid and incorporated their ideas in the lab report. 299 Students indicated as a good idea coating the sensor with a 300 Nafion film since it is a negatively charged polymer that repels 301 anions. $^{32,33}$

\section{Application to Real Samples}

303 The final aim of the biosensor developed here was to determine 304 glucose concentrations in real samples. Thus, students 305 determined glucose concentrations in a cola beverage and 306 orange juice purchased in the market. The only pretreatment 307 needed was dilution of the sample to obtain a concentration 308 within the linear range of the biosensor (dilutions were made in $3090.1 \mathrm{M}$ Tris- $\mathrm{HNO}_{3}$ buffer solution, $\mathrm{pH}$ 7.0). Previous degassing 310 by stirring was required for cola samples.

311 The samples were validated previously by the instructor 312 using an alternative method (a commercial glucose enzymatic 313 assay kit with spectrophotometric detection), and the results were given to the students after they analyzed the samples. In 314 this way, students compared the results obtained using the 315 biosensor with the results given by a "reference method". The 316 values for glucose concentration obtained with the sensor and 317 the commercial kit are summarized in Table 3. Students $318 \mathrm{t} 3$

Table 3. Application of the Biosensor to Analysis of Real Samples

\begin{tabular}{ccc} 
& \multicolumn{2}{c}{ Glucose Concentration Determination, $\mathrm{g} / 100 \mathrm{~mL}^{a}$} \\
\cline { 2 - 3 } Sample & Electrochemical Sensor & Spectrophotometric Assay \\
Cola beverage & $3.3 \pm 0.3$ & $3.42 \pm 0.03$ \\
Orange juice & $3.4 \pm 0.2$ & $3.47 \pm 0.04$
\end{tabular}

${ }^{a}$ Data are given as mean \pm SD calculated with two degrees of freedom and $p=0.05 ; n=3$.

statistically compared the mean values obtained using the two 319 methodologies through a Student's $t$ test. ${ }^{34}$ The $t$ values 320 calculated for the cola beverage and orange juice were less than 321 the $t$ value tabulated for two degrees of freedom and a 0.05322 significance level. Thus, there were no significant differences 323 between the glucose concentrations given by the biosensor and 324 the enzymatic assay.

\section{DISCUSSION}

After constructing the glucose biosensor, the students learned 327 about different electrochemical techniques and their application 328 to the development of an enzymatic biosensor. They also 329 realized that the use of low-cost, disposable, miniaturized, and 330 portable equipment is of paramount importance today, 331 especially when real samples are analyzed.

The experiment was completed with discussions on the 333 following topics:

(i) The analytical problem: types of samples and levels of 335 glucose.

(ii) The state of the art: previous works obtained from a 337 bibliographic search on glucose electrochemical enzy- 338 matic sensors, discussing also the different generations of 339 sensors, the role of nanomaterials, and nonenzymatic 340 approaches.

(iii) Evolution of electroanalysis from conventional cells to 342 miniaturized designs.

(iv) Analytical properties (accuracy, precision, sensitivity, 344 selectivity, and especially those related to productivity: 345 analysis of time and cost-see the Supporting 346 Information) and approaches for improving them.

\section{CONCLUSIONS}

This experiment served as a practical introduction to biosensor 349 technology and to the challenge of glucose determination. The 350 high number of diabetes patients worldwide increased its 351 relevance, and the application of the developed sensor in 352 analysis of real samples stimulated the students' interest. This 353 lab experiment also introduced students to the miniaturization 354 and simplification of analytical devices and methodologies, 355 some of the most important trends in modern analytical 356 chemistry. Biosensors are an excellent example of simple and 357 promising analytical tools, and students could become 358 familiarized with their two components (sensing zone and 359 transducer), understanding concepts of enzymatic analysis and 360 electroanalysis at the same time. Moreover, they learned how to 361 use screen-printed electrodes, which are widely used today in 362 
363 the development of sensors. They also discussed analytical 364 properties (e.g., accuracy, precision, sensitivity, and selectivity) 365 and productivity-related features (e.g., analysis time and cost). 366 In summary, this lab experiment allowed students to acquire 367 problem-solving skills, to reach a high level of critical thought, 368 and to be more confident in facing real-world analytical 369 problems.

\section{ASSOCIATED CONTENT}

\section{S Supporting Information}

372 The Supporting Information is available on the ACS 373 Publications website at DOI: 10.1021/acs.jchemed.6b00948.

$374 \quad$ Notes for instructors and a suggested student handout 375 (PDF, DOCX)

\section{AUTHOR INFORMATION}

377 Corresponding Authors

378 *E-mail: ecosta.rama@gmail.com.

$379 *$ E-mail: costa@uniovi.es.

380 ORCID $\odot$

381 A. Costa-García: 0000-0002-7630-1497

382 Notes

383 The authors declare no competing financial interest.

\section{ACKNOWLEDGMENTS}

385 This work was supported by the FC-15-GRUPIN-021 Project 386 from the Asturias Regional Government and the CTQ2014387 58826-R Project from the Spanish Ministry of Economy and 388 Competitiveness (MINECO).

\section{$389 \square$ REFERENCES}

390 (1) Valcárcel, M.; Cárdenas, S.; Gallego, M. Sample screening 391 systems in analytical chemistry. TrAC, Trends Anal. Chem. 1999, 18 392 (11), 685-694.

393 (2) Gałuszka, A.; Migaszewski, Z.; Namieśnik, J. The 12 principles of 394 green analytical chemistry and the significance mnemonic of green 395 analytical properties. TrAC, Trends Anal. Chem. 2013, 50, 78-84.

396 (3) Rackus, D. G.; Shamsi, M. H.; Wheeler, A. R. Electrochemistry, 397 biosensors and microfluidics: a convergence of fields. Chem. Soc. Rev. 398 2015, 44, 5320-5340.

399 (4) Taleat, Z.; Khoshroo, A.; Mazloum-Ardakani, M. Screen-printed 400 electrodes for biosensing: a review (2008-2013). Microchim. Acta 401 2014, 181 (9-10), 865-891.

402 (5) Domínguez Renedo, O.; Alonso-Lomillo, M. A.; Arcos Martínez, $403 \mathrm{M}$. J. Recent developments in the field of screen-printed electrodes 404 and their related applications. Talanta 2007, 73 (2), 202-219.

405 (6) Hayat, A.; Marty, J. L. Disposable screen printed electrochemical 406 sensors: tools for environmental monitoring. Sensors 2014, 14 (6), $407 \quad 10432-10453$.

408 (7) Jung, W.; Han, J.; Choi, J.; Ahn, C. H. Point-of-care testing 409 (POCT) diagnostic systems using microfluidic lab-on-a-chip tech410 nologies. Microelectron. Eng. 2015, 132, 46-57.

411 (8) Biosensors and Bioelectronics Web Page. http://www.journals. 412 elsevier.com/biosensors-and-bioelectronics/ (accessed February 413 2017).

414 (9) Galant, A. L.; Kaufman, R. C.; Wilson, J. D. Glucose: detection 415 and analysis. Food Chem. 2015, 188, 149-160.

416 (10) Heller, A.; Feldman, B. Electrochemical glucose sensors and 417 their applications in diabetes management. Chem. Rev. 2008, 108 (7), 418 2482-2505.

419 (11) American Diabetes Association. Standards of medical care in 420 diabetes-2014. Diabetes Care 2014, 37 (Suppl. 1), S14-S80.
(12) Wang, J. Electrochemical glucose biosensors. Chem. Rev. 2008, 421 108, 814-825.

(13) Wang, H.; Lee, A. Recent developments in blood glucose 423 sensors. J. Food Drug Anal. 2015, 23 (2), 191-200.

(14) Biscay, J.; Rama, E. C.; García, M. B. G.; Carrazón, J. M. P.; 425 García, A. C. Enzymatic sensor using mediator-screen-printed carbon 426 electrodes. Electroanalysis 2011, 23 (1), 209-214. 427

(15) Tothill, I. E. Biosensors developments and potential applications 428 in the agricultural diagnosis sector. Comput. Electron. Agric. 2001, 30, 429 205-218.

430

(16) Biscay, J.; Costa Rama, E.; González García, M. B.; Julio Reviejo, 431 A.; Pingarrón Carrazón, J. M.; García, A. C. Amperometric fructose 432 sensor based on ferrocyanide modified screen-printed carbon 433 electrode. Talanta 2012, 88, 432-438.

(17) Rama, E. C.; Biscay, J.; González García, M. B.; Reviejo, A. J.; 435 Pingarrón Carrazón, J. M.; Costa García, A. Comparative study of 436 different alcohol sensors based on screen-printed carbon electrodes. 437 Anal. Chim. Acta 2012, 728, 69-76.

438

(18) Blanco-López, M. C.; Lobo-Castañón, M. J.; Miranda-Ordieres, 439 A. J. Homemade bienzymatic-amperometric biosensor. J. Chem. Educ. 440 2007, 84 (4), 677-680.

(19) Choi, M. M. F.; Wong, P. S. Application of a datalogger in 442 biosensing: a glucose biosensor. J. Chem. Educ. 2002, 79 (8), 982.443 (20) Hobbs, J. M.; Patel, N. N.; Kim, D. W.; Rugutt, J. K.; Wanekaya, 444 A. K. Glucose determination in beverages using carbon nanotube 445 modified biosensor: an experiment for the undergraduate laboratory. J. 446 Chem. Educ. 2013, 90, 1222-1226. 447

(21) Ruzgas, T.; Csöregi, E.; Emnéus, J.; Gorton, L.; Marko-Varga, G. 448 Peroxidase-modified electrodes: fundamentals and application. Anal. 449 Chim. Acta 1996, 330 (2-3), 123-138.

(22) Gu, M.; Wang, J.; Tu, Y.; Di, J. Fabrication of reagentless 45 glucose biosensors: a comparison of mono-enzyme GOD and 452 bienzyme GOD-HRP systems. Sens. Actuators, B 2010, 148 (2), 453 486-491.

(23) Chaubey, A.; Malhotra, B. D. Mediated biosensors. Biosens. 455 Bioelectron. 2002, 17 (6-7), 441-456.

456

(24) Thiyagarajan, N.; Chang, J. L.; Senthilkumar, K.; Zen, J. M. 457 Disposable electrochemical sensors: a mini review. Electrochem. 458 Commun. 2014, 38, 86-90.

(25) Bard, A. J.; Faulkner, L. R. Electrochemical Methods: 460 Fundamentals and Applications, 2nd ed.; John Wiley \& Sons: New 461 York, 2001.

462

(26) Amor-Gutiérrez, O.; Rama, E. C.; Costa-García, A.; Fernández- 463 Abedul, M. T. Paper-based maskless enzymatic sensor for glucose 464 determination combining ink and wire electrodes. Biosens. Bioelectron. 465 2016. http://dx.doi.org/10.1016/j.bios.2016.11.008.10.1016/466 j.bios.2016.11.008

(27) Armbruster, D. A.; Pry, T. Limit of blank, limit of detection and 468 limit of quantitation. Clin. Biochem. Rev. 2008, 29 (Suppl.1), S49-S52. 469

(28) Shrivastava, A.; Gupta, V. Methods for the determination of 470 limit of detection and limit of quantitation of the analytical methods. 471 Chronicles Young Sci. 2011, 2 (1), 21.

(29) Lee, S. H.; Fang, H. Y.; Chen, W. C. Amperometric glucose 473 biosensor based on screen-printed carbon electrodes mediated with 474 hexacyanoferrate-chitosan oligomers mixture. Sens. Actuators, B 2006, 475 117 (1), 236-243.

476

(30) Luo, J.; Jiang, S.; Zhang, H.; Jiang, J.; Liu, X. A novel non- 477 enzymatic glucose sensor based on $\mathrm{Cu}$ nanoparticle modified graphene 478 sheets electrode. Anal. Chim. Acta 2012, 709, 47-53.

479

(31) Barberis, A.; Spissu, Y.; Fadda, A.; Azara, E.; Bazzu, G.; 480 Marceddu, S.; Angioni, A.; Sanna, D.; Schirra, M.; Serra, P. A. 481 Simultaneous amperometric detection of ascorbic acid and antioxidant 482 capacity in orange, blueberry and kiwi juice, by a telemetric system 483 coupled with a fullerene- or nanotubes-modified ascorbate subtractive 484 biosensor. Biosens. Bioelectron. 2015, 67, 214-223. 485 (32) Salimi, A.; Abdi, K.; Khayatian, G. R. Amperometric detection of 486 dopamine in the presence of ascorbic acid using a nafion coated glassy 487 carbon electrode modified with catechin hydrate as a natural 488 antioxidant. Microchim. Acta 2004, 144 (1), 161-169. 
490 (33) Jeong, H.; Jeon, S. Determination of dopamine in the presence 491 of ascorbic acid by nafion and single-walled carbon nanotube film 492 modified on carbon fiber microelectrode. Sensors 2008, 8 (11), 69244936935.

494 (34) Miller, J.; Miller, J. C. Statistics and Chemometrics for Analytical 495 Chemistry, 6th ed.; Prentice Hall: Harlow, U.K., 2010; pp 37-73. 